\title{
Lorentz violation with an invariant minimum speed as foundation of the uncertainty principle in Minkowski, dS and AdS spaces
}

\author{
Cláudio Nassif da Cruz \\ CPFT-MG:Centro de Pesquisas em Física Teórica-MG, \\ Rua Rio de Janeiro 1186, Lourdes, Belo Horizonte-MG, CEP:30.160-041, Brazil \\ claudionassif@yahoo.com.br
}

Received 7 February 2019

Revised 11 April 2019

Accepted 25 April 2019

Published 17 May 2019

\begin{abstract}
This research aims to provide the geometrical foundation of the uncertainty principle within a new causal structure of spacetime so-called Symmetrical Special Relativity (SSR), where there emerges a Lorentz violation due to the presence of an invariant minimum speed $V$ related to the vacuum energy. SSR predicts that a dS scenario occurs only for a certain regime of speeds $v$, where $v<v_{0}=\sqrt{c V}$, which represents the negative gravitational potentials $(\Phi<0)$ connected to the cosmological parameter $\Lambda>0$. For $v=v_{0}$, Minkowski (pseudo-Euclidean) space is recovered for representing the flat space $(\Lambda=0)$, and for $v>v_{0}(\Phi>0)$, Anti-de Sitter (AdS) scenario prevails $(\Lambda<0)$. The fact that the current universe is flat as its average density of matter distribution $\left(\rho_{m}\right.$ given for a slightly negative curvature $R$ ) coincides with its vacuum energy density ( $\rho_{\Lambda}$ given for a slightly positive curvature $\Lambda$ ), i.e. the cosmic coincidence problem, is now addressed by SSR. SSR provides its energy-momentum tensor of perfect fluid, leading to the EOS of vacuum $\left(p=-\rho_{\Lambda}\right)$. Einstein equation for vacuum given by such SSR approach allows us to obtain $\rho_{\Lambda}$ associated with a scalar curvature $\Lambda$, whereas the solution of Einstein equation only in the presence of a homogeneous distribution of matter $\rho_{m}$ for the whole universe presents a scalar curvature $R$, in such a way that the presence of the background field $\Lambda$ opposes the Riemannian curvature $R$, thus leading to a current effective curvature $R_{\text {eff }}=R+\Lambda \approx 0$ according to observations. This corrects the notion of gravity as being only of Riemannian origin as the flat space has connection with a background gravity. In view of the current dS scenario with a quasi-zero $\Lambda$ slightly larger than $|R|$, we will just obtain a Generalized Uncertainty Principle (GUP) given in the cases of weak gravity and anti-gravity.
\end{abstract}

Keywords: Lorentz violation; invariant minimum speed; generalized uncertainty principle; vacuum energy; dS and AdS spaces.

PACS Nos.: 11.30.Qc, 98.80.Qc

This is an Open Access article published by World Scientific Publishing Company. It is distributed under the terms of the Creative Commons Attribution 4.0 (CC-BY) License. Further distribution of this work is permitted, provided the original work is properly cited. 


\section{Introduction}

Lorentz violations can be observable in nature. ${ }^{1,2}$ In the last two decades, the physicists have shown a great interest in the theories that contained the breakdown of Lorentz symmetry in many scenarios ${ }^{3-13}$ and also the so-called Deformed Special Relativities (DSR), $\stackrel{14,15}{ }$ in spite of the fact that no relevant experimental fact has demonstrated the existence of a Lorentz violation until the present time. However, there could be the evidence that the Lorentz symmetry breaking may exist in a very low energy regime due to the presence of a vacuum energy density connected to the well-known cosmological constant $\Lambda$ related to a universal background field (a preferred reference frame) associated to an invariant minimum speed. So the effects of an invariant minimum speed on the breakdown of Lorentz symmetry at lower energies have already been exhaustively investigated by the so-called Symmetrical Special Relativity (SSR) theory, where there should be two invariant speeds, namely the well-known speed of light $c$ (the maximum speed) for much higher energies and an invariant minimum speed $V\left(\sim 10^{-14} \mathrm{~m} / \mathrm{s}\right)$ for much lower energies, leading to kinematic non-locality. $\underline{\underline{16}-24}$

Here, we should mention an interesting recent paper about SSR entitled "Lorentz violation with a universal minimum speed as foundation of de Sitter (dS) relativity", 19 where we have shown that the invariant minimum speed provides the foundation for understanding the conformal metric that represents the dS-relativity. So, we have explored the nature of the SSR-metric ${ }^{16}$ in order to understand the origin of the conformal factor that appears in the metric by deforming Minkowski metric by means of a scale factor $\Theta(v)^{19}$ depending on the minimum speed that breaks down the Lorentz symmetry, thus leading to a positive cosmological constant. We have also found that SSR-metric provides a set of infinite curvatures, $\frac{19}{}$ i.e. it behaves like an extended dS-relativity, where a strong anti-gravity can be also taken into account when $\Lambda$ is too large. In sum, we have shown that SSR-metric includes the following interval of $\Lambda$, namely $0<\Lambda<\infty$, such that we have an extended dS-relativity. Furthermore, we have also shown that SSR-metric is a solution of Einstein equation in a dS Scenario (with positive cosmological constant) governed by vacuum, ${ }^{19}$ i.e. in the absence of matter $\left(T_{\mu \nu}=0\right)$.

In this paper based on the previous one, ${ }^{19}$ thanks to the fact that it was already shown that SSR-metric is a solution of Einstein equation in dS scenario, it will be possible to use the energy-momentum tensor $T_{\mu \nu}^{\mathrm{vac}}=T_{\mu \nu}^{\mathrm{ssr}}$ of perfect fluid (vacuum) given by the 4 -velocities of SSR in the place of the usual $T_{\mu \nu}$ that presents the well-known 4-velocities with Lorentz invariance. So, we will go beyond by replacing Einstein equation with the cosmological constant and absence of matter by its equivalent form given in the SSR-vacuum, where the ad hoc term of cosmological constant is replaced by its equivalent tensor $T_{\mu \nu}^{\mathrm{ssr}}$ of vacuum with the 4 -velocities of SSR. ${ }^{16}$ After this, we will make the limit $v \rightarrow V$ in the second term of the equation according to SSR. This is exactly equivalent of making $p=-\rho$, i.e. the equation of state (EOS) of vacuum (the cosmological constant). 
Finally, by comparing such term of vacuum of SSR with the well-known ad hoc term of cosmological constant in dS scenario, we will find $\rho_{\Lambda}$, which is consistent with the result of the modern cosmology of an accelerated universe, where the

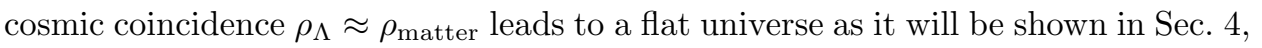
contrary to a Riemannian (curved) universe predicted by the standard cosmology. In this sense, we should realize that the geometrical foundations of the standard cosmology are not equivalent to the geometrical representations of the observations, because Riemannian geometry lacks some details in the description of the universe. In fact, it is notorious that there is a topological deficiency that cannot be ignored by Einstein's gravitational theory because the flat spacetime acts also as a ground state for gravitation and SSR is able to remove such lack, since the cosmological constant $\Lambda$ as an impediment to the existence of Minkowski spacetime is due to its direct connection with the minimum speed $V, \frac{16,17}{1}$ so that SSR spacetime (SSRconformal metric ${ }^{19}$ ) is a solution of Einstein's equation with the presence of $\Lambda$ and absence of matter, representing a new ground state of the gravitational field, i.e. the dS ground state.

In Sec. 2 , we will make a brief review of the transformations of spacetime in SSR. 16

In Sec. 3 , we will investigate the origin of the Uncertainty Principle and the undulatory nature of matter within the SSR scenario.

In Sec. 5 , we will make a generalization of the Uncertainty Principle (GUP) in $\mathrm{dS}$ and AdS spaces, where just a weak gravity and anti-gravity due to a tiny positive cosmological constant will be taken into account as the current universe is practically flat according to the $\Lambda \mathrm{CDM}$ model, so that we are just interested to investigate GUP close to such $\Lambda \mathrm{CDM}$ scenario.

\section{Transformations of Spacetime in SSR}

The $(1+1) D$-transformations in $\operatorname{SSR}^{16-22}$ with $\mathbf{v}=v_{x}=v$ (Fig. 1 ) are

$$
x^{\prime}=\Psi(X-v t+V t)=\theta \gamma(X-v t+V t)
$$

and

$$
t^{\prime}=\Psi\left(t-\frac{v X}{c^{2}}+\frac{V X}{c^{2}}\right)=\theta \gamma\left(t-\frac{v X}{c^{2}}+\frac{V X}{c^{2}}\right),
$$

where the factor $\theta=\sqrt{1-V^{2} / v^{2}}$ and $\Psi=\theta \gamma=\sqrt{1-V^{2} / v^{2}} / \sqrt{1-v^{2} / c^{2}}$.

The $(3+1) D$-transformations in SSR were shown in a previous paper ${ }^{16}$ as follows:

$$
\mathbf{r}^{\prime}=\theta\left[\mathbf{r}+(\gamma-1) \frac{(\mathbf{r} \cdot \mathbf{v})}{v^{2}} \mathbf{v}-\gamma \mathbf{v}(1-\alpha) t\right]
$$

where $\alpha=V / v$.

And

$$
t^{\prime}=\theta \gamma\left[t-\frac{\mathbf{r} \cdot \mathbf{v}}{c^{2}}(1-\alpha)\right]
$$




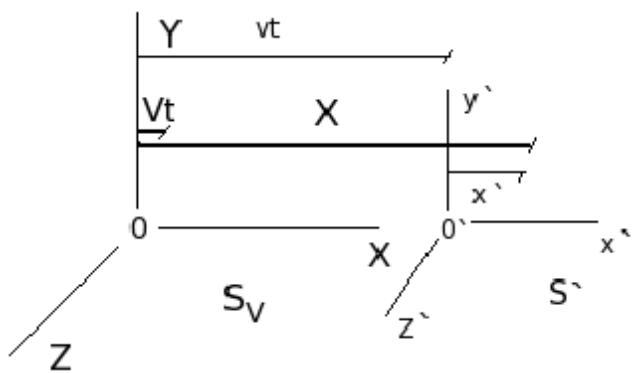

Fig. 1. In this special case $(1+1) D$, the referential $S^{\prime}$ moves in $x$-direction with a speed $v(>V)$ with respect to a preferred reference frame connected to the ultra-referential $S_{V}$ (background field). If $V \rightarrow 0, S_{V}$ is eliminated and thus the Galilean frame $S$ takes place, recovering the Lorentz transformations.

From Eqs. (3) and (4), we can verify that, if we consider $\mathbf{v}$ to be in the same direction of $\mathbf{r}$, with $r=X$, we recover the special case of $(1+1) D$-transformations given by Eqs. (1) and (2).

The metric of SSR is a Minkowski metric deformed by a multiplicative function, i.e. a scale factor $\Theta(v)$ with $v$-dependence. $\Theta(v)$ works like a conformal factor, which leads to a kind of dS-metric, ${ }^{19}$ namely $d \mathcal{S}^{2}=\Theta \eta_{\mu \nu} d x^{\mu} d x^{\nu}$, where $\Theta=\Theta(v)=$ $\theta^{-2}=1 /\left(1-V^{2} / v^{2}\right) \equiv 1 /\left(1-\Lambda r^{2} / 6 c^{2}\right)^{2}$ (Ref. 19) is the conformal factor and $\eta_{\mu \nu}$ is the well-known Minkowski metric.

\section{Geometrization of the Fundamental Quantum Phenomena Within SSR Scenario}

This section aims to investigate the geometrical origin of the Uncertainty Principle ${ }^{22}$ and the undulatory nature of matter within a quantum Machian scenario where the vacuum energy of the background framework $S_{V} \underline{16}$ plays a fundamental role in understanding the inertial mass of a particle, which has origin in its interaction with the whole universe by means of the vacuum- $S_{V}$. In this sense, we should consider such interaction as being related to a quantum Machian scenario generated by the spacetime of SSR.

\subsection{The concepts of reciprocal 4-velocity and reciprocal 4-momentum}

The idea of reciprocal speed $v_{\text {rec }}=v_{0}^{2} / v=c V / v$ (Refs. 22-24) allows us to build the contravariant and covariant 4-reciprocal velocities. As we already know that the contravariant and covariant 4 -velocities are $\mathcal{U}^{\mu}=\Psi(v)\left[1, v_{\alpha} / c\right]$ and $\mathcal{U}_{\mu}=\Psi(v)\left[1,-v_{\alpha} / c\right], \frac{16}{,}$ we simply write the contravariant and covariant 4-reciprocal velocities in the following way:

$$
\mathcal{U}_{\mathrm{rec}}^{\mu}=\Psi\left(v_{\mathrm{rec}}\right)\left[1, \frac{v_{\mathrm{rec}, \alpha}}{c}\right]=\left[\frac{\sqrt{1-\frac{V^{2}}{v_{\mathrm{rec}}}}}{\sqrt{1-\frac{v_{\mathrm{rec}}^{2}}{c^{2}}}}, \frac{v_{\mathrm{rec}, \alpha} \sqrt{1-\frac{V^{2}}{v_{\mathrm{rec}}^{2}}}}{c \sqrt{1-\frac{v_{\mathrm{rec}}^{2}}{c^{2}}}}\right]
$$


and

$$
\mathcal{U}_{\mu, \mathrm{rec}}=\Psi\left(v_{\mathrm{rec}}\right)\left[1,-\frac{v_{\mathrm{rec}, \alpha}}{c}\right]=\left[\frac{\sqrt{1-\frac{V^{2}}{v_{\mathrm{rec}}^{2}}}}{\sqrt{1-\frac{v_{\mathrm{rec}}^{2}}{c^{2}}}},-\frac{v_{\mathrm{rec}, \alpha} \sqrt{1-\frac{V^{2}}{v_{\mathrm{rec}}^{2}}}}{c \sqrt{1-\frac{v_{\mathrm{rec}}^{2}}{c^{2}}}}\right],
$$

where $\mu=0,1,2,3$ and $\alpha=1,2,3$. We find $v_{\text {rec }}^{2} / c^{2}=V^{2} / v^{2}$ and $V^{2} / v_{\text {rec }}^{2}=v^{2} / c^{2}, 22$ so that we have $V<v<c$ and $V<v_{\text {rec }}<c$, where $V$ is the reciprocal of $c$ and vice versa.

So, now by substituting $v_{\text {rec }}=v_{0}^{2} / v=c V / v$ in Eqs. (5) and (6) and after performing the calculations, we finally obtain the contravariant and covariant 4reciprocal velocities in function of $v$, namely:

$$
\mathcal{U}_{\text {rec }}^{\mu}=\Psi(v)^{-1}\left[1, \frac{V}{v_{\alpha}}\right]
$$

and

$$
\mathcal{U}_{\mu, \mathrm{rec}}=\Psi(v)^{-1}\left[1,-\frac{V}{v_{\alpha}}\right]
$$

where $\Psi(v)^{-1}=\sqrt{1-v^{2} / c^{2}} / \sqrt{1-V^{2} / v^{2}}$.

It is important to note that all the components of both contravariant and covariant 4-reciprocal velocities diverge when the speed $v$ is closer to the minimum speed $V$, i.e. $v \rightarrow V$, thus leading to $\mathcal{U}_{\text {rec }}^{\mu}(V)=[\infty, \infty]$ and $\mathcal{U}_{\mu, \text { rec }}(V)=[\infty,-\infty]$, while, on the other hand, the components of both contravariant and covariant 4-velocities become zero, i.e. $\mathcal{U}^{\mu}(V)=\mathcal{U}_{\mu}(V)=[0,0]$. Thus, we realize that the reciprocal 4velocity represents exactly the inverse of the 4 -velocity in the limit $v \rightarrow V$. This leads us to strengthen the conclusion that the spacetime of SSR naturally contains a quantum aspect of non-locality given by an "internal" motion $\left(\mathcal{U}_{\text {rec }}^{\mu}\right)$ of the particle that diverges when its momentum tends to zero.

We will show that the divergence of the reciprocal 4 -velocity $\mathcal{U}_{\text {rec }}^{\mu}$ so close to the minimum speed $V$ is directly related to a complete delocalization of the particle when its momentum goes to zero so close to $V$. This means the increasing of the uncertainty on position of a particle when the uncertainty on momentum tends to zero, according to the Uncertainty Principle, but this subject will be deeper investigated soon.

The contravariant and covariant reciprocal 4-momenta are given as follows:

$$
\mathcal{P}_{\text {rec }}^{\mu}=m_{0} c \mathcal{U}_{\text {rec }}^{\mu}=m_{0} c \Psi(v)^{-1}\left[1, \frac{V}{v_{\alpha}}\right]
$$

and

$$
\mathcal{P}_{\mu, \mathrm{rec}}=m_{0} c \mathcal{U}_{\mu, \mathrm{rec}}=m_{0} c \Psi(v)^{-1}\left[1,-\frac{V}{v_{\alpha}}\right] .
$$

Here it must be stressed that the reciprocal 4-momentum has an important physical meaning. So, in order to perceive such a new physical meaning, let us 
make the vacuum approximation $v \approx V$ for the reciprocal 4-momentum and so we obtain

$$
\mathcal{P}_{\text {rec }}^{\mu}(v \approx V)=\left[\frac{m_{0} c}{\sqrt{1-\frac{V^{2}}{v^{2}}}}, \frac{m_{0}}{\sqrt{1-\frac{V^{2}}{v^{2}}}}\left(\frac{c V}{v}\right)\right],
$$

where $c V / v=v_{0}^{2} / v=v_{\text {rec }}$ is the reciprocal speed..$^{22}$

We should note that the mass $m_{\text {dress }}=m_{0} / \sqrt{1-V^{2} / v^{2}}$ (Ref. 17) that appears in the components of the reciprocal 4-momentum for the approximation $v \approx V$ [Eq. (11)] is already known as being the dressed mass that increases to infinite in the limit $v \rightarrow \infty$. This means that the particle becomes strongly coupled to the background field of the whole universe (vacuum energy) within a so-called quantum Machian scenario, $\stackrel{24}{r}$ thus leading to a drastic increasing of its inertial (effective) mass by working like a dressed mass, since the particle "diffuses" for being delocalized in the whole space in such a limit of $v \rightarrow V$. Thus, the reciprocal 4momentum in Eq. (11) should be denominated as the dressed 4-momentum written simply as follows:

$$
\mathcal{P}_{\text {rec }}^{\mu}(v \approx V)=\left[m_{\mathrm{dress}} c, m_{\mathrm{dress}} v_{\mathrm{rec}}\right],
$$

where the component $P_{\text {dress }}=m_{\text {dress }} v_{0}^{2} / v=m_{\text {dress }} v_{\text {rec }}$ represents the dressed (spatial) momentum of the particle close to the minimum speed $V$ (vacuum regime).

The components of temporal and spatial momenta in Eq. (12) assume very large values close to $V$, as the dressed mass $m_{\text {dress }}$ becomes too large. However, here it must be stressed that both components in Eqs. (12) or Eq. (11) become equal to each other when $v$ is much closer to the minimum speed $V$, that is to say when we make $v \rightarrow V$, we find $v_{\text {rec }}=c$, such that both components of $\mathcal{P}_{\text {rec }}^{\mu}$ become equal to $m_{\mathrm{rec}} c$. So we can accurately write the following limit:

$$
\mathcal{P}_{\text {rec }}^{\mu}(v \rightarrow V)=\left[m_{\text {dress }} c, m_{\text {dress }} c\right] \rightarrow[\infty, \infty] .
$$

Equation (13) shows two fundamental features that describe the particles of vacuum, namely:

(a) As both components of the dressed 4-momentum are in equal-footing, i.e. $P_{\text {dress }}^{0}=P_{\text {dress }}=m_{\text {dress }} c$, we conclude that the vacuum is made up of extremely giant bosons that carry very high momenta as their masses are too large.

(b) The vacuum is isotropic and the masses of the moving particles have origin in their interactions with such bosons that are extremely giant, in such a way that, only for $v \gg V$, the couplings of the particles with the background bosons become weaker and so the particles recover their finite masses $m_{0}$, since we have $m_{\mathrm{dress}}=m_{0} / \sqrt{1-V^{2} / v^{2}} \approx m_{0}$ for $V / v \approx 0$ or then $v \gg V$.

It is known that the Standard Model (SM) predicts the Higgs bosons that are very heavy $\left(\approx 125 \mathrm{GeV} / c^{2}\right)$ and they provide the masses of the particles. However, 
SM does not include gravity and that is the intriguing question still unsolved. As the inertial masses are equivalent to the gravitational masses, we expect that the most fundamental vacuum should have a gravitational origin in order to provide the masses of the particles within a Machian scenario, which is not taken into account in SM. In this sense, it seems that SM is not still the most fundamental theory to explain the origin of the masses as gravity is directly related to the inertia. Thus a theory beyond SM is needed to give us a more fundamental explanation for the origin of inertia. Equation (13) is beyond SM as it provides extremely heavy bosons whose masses can be much larger than the mass of Higgs boson. Such extremely heavy bosons are due to the existence of a gravitational vacuum 16 associated with a background field that breaks Lorentz symmetry within a Machian scenario. ${ }^{24}$ Here is the novelty that is beyond SM. So we hope LHC be able to produce bosons much heavier than the Higgs bosons at much higher energies. This is a great challenge that aims to search for the incompleteness of SM due to the background field of SSR by breaking Lorentz symmetry.

\subsection{Geometrical description of the uncertainty principle within SSR scenario}

Before making the geometrical description of the Uncertainty Principle, let us rewrite the four 4-vectors, as follows:

$$
\begin{aligned}
\mathcal{P}^{\mu} & =m_{0} c \Psi(v)\left[1, \frac{v_{\alpha}}{c}\right] ; \\
\mathcal{P}_{\mu} & =m_{0} c \Psi(v)\left[1,-\frac{v_{\alpha}}{c}\right] ; \\
\mathcal{U}_{\mathrm{rec}}^{\mu} & =\Psi(v)^{-1}\left[1, \frac{V}{v_{\alpha}}\right]
\end{aligned}
$$

and

$$
\mathcal{U}_{\mu, \mathrm{rec}}=\Psi(v)^{-1}\left[1,-\frac{V}{v_{\alpha}}\right],
$$

where $\mu=0,1.2,3$ and $\alpha=1,2,3$.

By first making the scalar product of Eq. (15) by Eq. (16), we obtain

$$
\mathcal{P}_{\mu} \mathcal{U}_{\text {rec }}^{\mu}=m_{0} c\left(1-\frac{V}{c}\right) .
$$

As the ratio $V / c=\xi\left(\sim 10^{-22}\right)^{16}$ is too small, we can neglect $\xi(\ll 1)$ in Eq. (18) and simply write

$$
\mathcal{P}_{\mu} \mathcal{U}_{\text {rec }}^{\mu}=m_{0} c
$$

Now it is important to notice that each particle with mass $m_{0}$ has its own reduced Compton wavelength $\star_{C}$, such that the universal reduced Planck constant $\hbar$ can be always written in the following way:

$$
m_{0} c \star_{C}=\hbar,
$$


where $m_{0}$ and $\star_{C}$ represent, respectively, the mass and the reduced Compton wavelength of any particles (e.g. for the electron, we have $m_{0}=m_{e} \sim 10^{-30} \mathrm{Kg}$ with $\left.\star_{C}=\star_{C e} \sim 10^{-12} \mathrm{~m}\right)$.

So, according to Eq. (20), by multiplying both terms of Eq. (19) by $\star_{C}$, we find

$$
\mathcal{P}_{\mu}\left(\star_{C} \mathcal{U}_{\text {rec }}^{\mu}\right)=\hbar
$$

From Eq. (21), we can take the following reciprocal contravariant 4-vector, namely:

$$
\mathcal{X}_{\text {rec }}^{\mu}=\star_{C} \mathcal{U}_{\text {rec }}^{\mu}
$$

In order to understand the important meaning of the 4-vector Eq. (22), let us first substitute Eq. (16) in Eq. (22) and so we write

$$
\mathcal{X}_{\mathrm{rec}}^{\mu}=\frac{\sqrt{1-\frac{v^{2}}{c^{2}}}}{\sqrt{1-\frac{V^{2}}{v^{2}}}}\left[\star_{C}, \frac{V}{v_{\alpha}} \star_{C}\right] \text {. }
$$

As the components of $\mathcal{X}_{\text {rec }}^{\mu}$ [Eq. (23)] have dimension of length, then its components provide a deformation of wavelength that goes to the infinite when $v \rightarrow V$, i.e. $\mathcal{X}_{\text {rec }}^{\mu}(V)=[\infty, \infty]$. Thus, we conclude that such 4 -vector presents quantum nature by representing exactly a complete delocalization of the particle (uncertainty on position) close to $V$ when the components of its 4-momentum $\mathcal{P}_{\mu}$ go to zero in such a limit, i.e. $\mathcal{P}_{\mu}(V)=[0,0]$. In view of this, the 4 -vector in Eq. (23) is denominated as the delocalization 4-vector in SSR spacetime.

On the other hand, Eq. (23) shows that the particle becomes much better located when $v$ is too close to $c$, i.e. $\mathcal{X}_{\text {rec }}^{\mu}(c)=[0,0]$, while $\mathcal{P}_{\mu}(c)=[\infty,-\infty]$ in Eq. (15).

As we have shown that Eq. (23) represents the delocalization 4-vector associated with the uncertainty on position, Eq. (21) is simply written as

$$
\mathcal{P}_{\mu} \mathcal{X}_{\text {rec }}^{\mu}=\hbar
$$

which is the 4-vector form of the momentum-position uncertainty in SSR spacetime.

We can alternatively get Eq. (24) within a metric form by taking into account the metric $\eta^{\mu \nu}=(1,-1,-1,-1)$, so that we write

$$
\eta^{\mu \nu} \mathcal{P}_{\mu} \mathcal{X}_{\nu, \text { rec }}=\hbar
$$

Second, by making the scalar product of Eq. (14) by Eq. (17), we obtain essentially the same previous results, but now having changed the signal in the spatial components of the 4 -vectors since we simply change contravariant vector by the covariant vector and vice versa. Thus we find

$$
\mathcal{P}^{\mu}\left(\lambda_{C} \mathcal{U}_{\mu, \mathrm{rec}}\right)=\hbar
$$

from where we take the reciprocal covariant 4-vector (delocalization 4-vector), namely:

$$
\mathcal{X}_{\mu, \text { rec }}=\star_{C} \mathcal{U}_{\mu, \text { rec }}
$$


So by substituting Eq. (17) in Eq. (27), now we obtain the delocalization 4-vector in its covariant form as follows:

$$
\mathcal{X}_{\mu, \mathrm{rec}}=\frac{\sqrt{1-\frac{v^{2}}{c^{2}}}}{\sqrt{1-\frac{V^{2}}{v^{2}}}}\left[\star_{C},-\frac{V}{v_{\alpha}} \star_{C}\right]
$$

so that we simply write Eq. (26) as follows:

$$
\mathcal{P}^{\mu} \mathcal{X}_{\mu, \text { rec }}=\hbar
$$

which is essentially the same result given in Eq. (24), but we just change the covariant form by the contravariant one and vice versa.

Now we can also get Eq. (29) within a metric form by considering the covariant metric $\eta_{\mu \nu}=(1,-1,-1,-1)$, so that we write

$$
\eta_{\mu \nu} \mathcal{P}^{\mu} \mathcal{X}_{\text {rec }}^{\nu}=\hbar
$$

Equations (30) and (25) represent the same relation, but given in its covariant and contravariant forms.

It is interesting to note that the energy-time uncertainty relation contains only scalar quantities. Such uncertainty relation naturally appears inside Eq. (24) or Eq. (29), written in the following alternative way:

$$
\mathcal{P}_{\mu} \mathcal{X}_{\text {rec }}^{\mu}=\mathcal{P}^{\mu} \mathcal{X}_{\mu, \text { rec }}=m_{0} c \star_{C}=m_{0} c^{2} \Psi\left(\frac{\star_{C}}{c}\right) \Psi^{-1}=\hbar,
$$

where $E=m_{0} c^{2} \Psi$ and $\star_{C} / c=t_{C}$ is the reduced Compton time with which the speed of light takes to travel the reduced Compton length of the particle.

From Eq. (31), if we make $v \rightarrow V$, we find an infinitely deformed time $\tau=$ $t_{C} \Psi^{-1}(v \approx V) \rightarrow \infty$, such that we obtain $E=m_{0} c^{2} \Psi(v \approx V) \rightarrow 0$. On the other hand, for $v \rightarrow c$, we find $\tau \rightarrow 0$ and $E \rightarrow \infty$.

So, from Eq. (31), let us get the following energy-time relation:

$$
E \tau=\hbar,
$$

where $E=E_{0} \Psi$ and $\tau=t_{C} \Psi^{-1}$, with $t_{C}=\star_{C} / c$.

It is very important to stress that the speed $v$ in all the equations of SSR is given with respect to a preferred reference frame $S_{V}$ related to the unattainable minimum speed $V$ (Fig. 1). So, all those equations are observer-independent as there is no observer at $S_{V}$. This is the reason why the relations given in Eqs. (24), (25), (29), (30) and (32) provide exactly the minimum value of "uncertainty", i.e. the reduced Planck constant $\hbar$. Actually, the constant $\hbar$ represents a minimum quantum action inherent in SSR spacetime, working like an intrinsic "uncertainty", which is observer-independent within an objective reality. However, when a classical observer is taken into account at any Galilean (inertial) reference frame $S$ $(v=0)$, it was already shown that both energy $E$ and momentum $P$ with respect to $S_{V}$ (no observer) are interpreted as uncertainties $\Delta E$ and $\Delta p$ at any Galilean framework, i.e. we have shown that $E \equiv \Delta E$ and $P \equiv \Delta p \cdot \frac{22}{}$ It was also shown 
that $X \equiv \Delta x$ and $\tau \equiv \Delta t,{ }^{22}$ so that Eq. (24) or Eqs. (29) and (32) given with respect to $S_{V}$ are interpreted as the minimum uncertainties of momentum-position and energy-time for a classical observer at a Galilean reference frame $S$, written as follows:

$$
\left[\mathcal{P}_{\mu} \mathcal{X}_{\mathrm{rec}}^{\mu}\right]_{S_{V}}=\left[\mathcal{P}^{\mu} \mathcal{X}_{\mu, \mathrm{rec}}\right]_{S_{V}} \equiv[\Delta p \Delta x]_{S}=\hbar
$$

and

$$
[E \tau]_{S_{V}} \equiv[\Delta E \Delta t]_{S}=\hbar
$$

We should realize that Eqs. (33) and (34) do not still take into account the presence of an observer that try to measure the quantities $P, X, E$ and $\tau$ by emitting a photon that transfers momentum and energy to the particle, so that there emerges an increasing of the uncertainties of such quantities, thus leading to the well-known inequalities, namely $\Delta p \Delta x \geq \hbar$ and $\Delta E \Delta t \geq \hbar$.

\subsection{The concept of reciprocal metric in SSR and its connection with the undulatory nature of particles}

When performing the scalar product between the delocalization 4-vectors given in Eqs. (23) and (28), we find

$$
\mathcal{X}_{\mathrm{rec}}^{\mu} \mathcal{X}_{\mu, \mathrm{rec}}=\star_{C}^{2}\left(1-\frac{v^{2}}{c^{2}}\right),
$$

or then

$$
\frac{1}{\left(1-\frac{v^{2}}{c^{2}}\right)} \eta^{\mu \nu} \mathcal{X}_{\mu, \mathrm{rec}} \mathcal{X}_{\nu, \mathrm{rec}}=\star_{C}^{2}
$$

It is very important to realize that Eq. (36) represents the SSR reciprocal metric. The meaning of the reciprocal metric is the great novelty of SSR as it provides a geometrical description of the undulatory nature of particles by means of their delocalization 4-vectors, and where the squares of their Compton wavelengths are invariant as well as is the square of spacetime interval $\left(S^{2}\right)$ in SSR metric, i.e.

$$
\frac{1}{\left(1-\frac{V^{2}}{v^{2}}\right)} \eta^{\mu \nu} \mathcal{X}_{\mu} \mathcal{X}_{\nu}=S^{2},
$$

where $\mathcal{X}_{\mu}$ is the position 4 -vector.

As we already know that the metric tensor of SSR in Eq. (37) is $G^{\mu \nu}=[1 /(1-$ $\left.\left.V^{2} / v^{2}\right)\right] \eta^{\mu \nu}$, it is interesting to verify that the reciprocal metric tensor is written as

$$
G_{\mathrm{rec}}^{\mu \nu}=\frac{1}{\left(1-\frac{V^{2}}{v_{\mathrm{rec}}^{2}}\right)} \eta^{\mu \nu}=\frac{1}{\left(1-\frac{v^{2}}{c^{2}}\right)} \eta^{\mu \nu},
$$

where we have $v_{\text {rec }}=(c V) / v$. 
We should note that the reciprocal metric obtained in Eq. (38) is in fact the own reciprocal metric given in Eq. (36), which is already expected. So let us write Eq. (36) as follows:

$$
\star_{C}^{2}=G_{\text {rec }}^{\mu \nu} \mathcal{X}_{\mu, \text { rec }} \mathcal{X}_{\nu, \text { rec }}
$$

which could be denominated as the undulatory metric equation of a particle.

Until now we have obtained three fundamental metric equations of SSR, namely the first equation is Eq. (25) [or Eq. (30)] that provides the geometrical description of the Uncertainty Principle; the second one is Eq. (36) [or Eq. (39)] that represents the undulatory metric equation of a particle by providing the geometrical description of the wave-particle duality associated with de Broglie relation and the third one is Eq. (37) that represents the conformal metric of SSR.

Finally the fourth metric equation can be easily obtained from Eq. (24) [or Eq. (29)] by changing the non-reciprocal quantity by its reciprocal form and vice versa. To do that, we must use Eq. (9) [or Eq. (10)], and so we write

$$
\mathcal{P}_{\text {rec }}^{\mu} \mathcal{X}_{\mu}=m_{0} c \Psi(v)^{-1} \Psi(v)(1, V / v)\left(\begin{array}{c}
c t \\
-X
\end{array}\right),
$$

from where we find

$$
\mathcal{P}_{\text {rec }}^{\mu} \mathcal{X}_{\mu}=m_{0} c\left(c t-\frac{V X}{v}\right)=m_{0} c^{2} t\left(1-\frac{V X}{v c t}\right) .
$$

But if we admit $t=t_{C}=\star_{C} / c$ in Eq. (41), we would obtain

$$
\mathcal{P}_{\text {rec }}^{\mu} \mathcal{X}_{\mu}=\hbar\left(1-\alpha \frac{X}{\star_{C}}\right),
$$

where we have $\hbar=m_{0} c_{C}$ and $\alpha=V / v$.

Equation (42) can be written in the metric form, namely:

$$
\eta_{\mu \nu} \mathcal{P}_{\text {rec }}^{\mu} \mathcal{X}^{\nu}=\hbar\left(1-\alpha \frac{X}{\star_{C}}\right)
$$

Equation (43) is another novelty and it leads to a deformed uncertainty relation for the reciprocal momentum as the reduced Planck constant is corrected by $v$ and $X$. Although this result deserves to be deeply investigated elsewhere, here let us search for some interpretations of Eq. (43) by first taking into account lower energies when the speed is close to $V$ (vacuum), i.e. $v \approx V$. In this regime, $\mathcal{P}_{\text {rec }}^{\mu}$ is the dressed 4-momentum [Eq. (11)] that diverges when $v \rightarrow V$ as the particle becomes strongly coupled to vacuum at much lower energies. So, by considering $\alpha \approx 1(v \approx V)$ in Eq. (43), we find

$$
\eta_{\mu \nu} \mathcal{P}_{\text {rec }}^{\mu}(v \approx V) \mathcal{X}^{\nu}=\hbar\left(1-\frac{X}{\hbar_{C}}\right),
$$

where we must have $X \leq c t_{C}\left(=\star_{C}\right)$ in such a regime. If $X=c t_{C}$, we would have a light-like interval as $X_{\mu} X^{\mu}=X^{2}-c^{2} t_{C}^{2}=0$. In this very special case of so low 
energies and with all the points connected by light inside a sphere of radius $\lambda_{C}$, the uncertainty relation for the dressed momentum is completely violated, so that we find

$$
\eta_{\mu \nu} \mathcal{P}_{\text {rec }}^{\mu}(v \approx V) \mathcal{X}^{\nu}=0
$$

Equation (45) shows us a particle with so low energy and a too high dressed momentum strongly confined by vacuum inside a very small sphere of radius $\lambda_{C}$. This special phenomenon of confinement could be understood as a quark confinement at too low energies inside the proton (QCD at ultra-infrared regime), where the proton radius $\left(r_{p} \sim 10^{-15} \mathrm{~m}\right)$ is exactly in the order of magnitude of the reduced Compton wavelength $\left(\star_{C q}\right)$ for the constituent mass of each quark, namely $m_{q}=m_{\text {quark }}=(1 / 3) m_{p} \sim 10^{-27} \mathrm{Kg}$, so that we indeed verify that $\star_{C q}=\hbar / m_{q} c \sim r_{p} \sim 10^{-15} \mathrm{~m}$.

Thus Eq. (45) shows us that, when the quark becomes even much more confined inside the proton just for $v \approx V$, the uncertainty relation is violated in such a way that we could localize the quark inside the proton.

A deeper investigation of the implications of Eq. (43) could show a similarity between QCD-vacuum and the gravitational vacuum within a Machian scenario where the whole universe works like a kind of a big bag inside which each particle presents a certain degree of confinement given by the reciprocal 4-momentum. Such similarity between a microscopic confinement system (e.g. quarks in the proton) and the cosmological one (particles in the universe) leads to the so-called holography.

Other cases emerge from Eq. (43), as for instance, the case of $\alpha \ll 1(v \gg V)$ or even for $v \rightarrow c$ when the particle becomes unconfined, since $\mathcal{P}_{\text {rec }}^{\mu}(v \approx c) \approx 0$. So, due to many interpretations emerging from Eq. (43), such broad issue will be explored elsewhere.

\section{The Flat Universe and the Problem of Cosmic Coincidence Under SSR}

\subsection{The cosmological constant as a positive curvature (cosmological anti-gravity) and its connection with the minimum speed as foundation of the EOS of vacuum}

It is already known that the current universe has a very low average density of matter $\rho_{m}$ that mysteriously coincides with its vacuum energy density $\rho_{\Lambda}$, i.e. $\rho_{m} \approx \rho_{\Lambda} \sim 10^{-29} \mathrm{~g} / \mathrm{cm}^{3}$. This is the well-known cosmic coincidence problem, which is due to the presence of the tiny value of the cosmological constant $\Lambda \sim 10^{-35} \mathrm{~s}^{-2}$ that leads to a cosmological anti-gravity whose repulsive effect counterposes exactly the attractive gravity of the very low density of matter $\rho_{m}$, in such a way that the geometry of the universe becomes quasi-flat according to observations. In this sense the presence of the cosmological constant corrects the topological deficiency of Einstein equation as the flat space now can be considered a ground state of grav- 
itational field, contrary to Minkowski (flat) space as being a ground state without gravity.

In this section, we show how the cosmological constant implements such new gravitational ground state in the Einstein equation by means of SSR theory. To do that, we should take into account the energy-momentum tensor of perfect fluid of SSR $\left(T_{\mu \nu}^{\mathrm{ssr}}\right)$ already investigated in a previous work, $\frac{16}{16}$ where we have defined

$$
T_{\mu \nu}^{\mathrm{ssr}}=(p+\rho) \mathcal{U}_{\mu} \mathcal{U}_{\nu}-p g_{\mu \nu},
$$

where $p$ is the pressure, $\rho$ being the energy density and $\mathcal{U}_{\mu}=\Psi(v)\left[1,-v_{\alpha} / c\right](\alpha=$ $1,2,3)$ is the covariant 4 -velocity of the SSR theory, where we already know that $\Psi(v)=\sqrt{1-V^{2} / v^{2}} / \sqrt{1-v^{2} / c^{2}}$.

We should have in mind that such energy-momentum tensor given in the limit of vacuum of SSR, i.e. $v \rightarrow V$, leads to $T_{\mu \nu, \mathrm{vac}}^{\mathrm{ssr}}=-p g_{\mu \nu}$, where the first term of 4 -velocities vanishes as we find $\mathcal{U}_{\mu}(v \rightarrow V)=0$. Besides this, we note that when considering $p=-\rho$, this leads to the same result, namely:

$$
T_{\mu \nu, \mathrm{vac}}^{\mathrm{ssr}}=-p g_{\mu \nu}=\rho g_{\mu \nu},
$$

where we realize that the EOS of vacuum $\left(p=-\rho=-\rho_{\Lambda}\right)$ emerges naturally from the spacetime of SSR in the sense that $T_{\mu \nu \text {,vac }}^{\mathrm{ssr}}$ given in the limit $V$ plays the same role of the cosmological constant $\Lambda$ placed into the Einstein equation in ad hoc way for representing the dS scenario. Thus, according to SSR, we write the Einstein equation in a dS scenario (also in the absence of matter), namely:

$$
R_{\mu \nu}-\frac{1}{2} R g_{\mu \nu}=\frac{8 \pi G}{c^{2}}\left[\lim _{v \rightarrow V} T_{\mu \nu}^{\mathrm{ssr}}\right]
$$

which leads to

$$
R_{\mu \nu}-\frac{1}{2} R g_{\mu \nu}=\frac{8 \pi G}{c^{2}} T_{\mu \nu, \mathrm{vac}}^{\mathrm{ssr}}=\frac{8 \pi G}{c^{2}} \rho_{\Lambda} g_{\mu \nu}
$$

or simply

$$
R_{\mu \nu}-\frac{1}{2} R g_{\mu \nu}-\frac{8 \pi G}{c^{2}} \rho_{\Lambda} g_{\mu \nu}=0
$$

with $\rho_{\Lambda}=-p$.

On the other hand, we already know the Einstein equation in the absence of matter and with cosmological constant in a dS scenario, as follows:

$$
R_{\mu \nu}-\frac{1}{2} R g_{\mu \nu}-\Lambda g_{\mu \nu}=0
$$

Finally, by comparing Eq. (51) above with its equivalent form [Eq. (50)] given in the vacuum of SSR, whose EOS is the same of the cosmological constant $\left(p=-\rho_{\Lambda}\right)$, we obtain

$$
\rho_{\Lambda}=-p=\frac{\Lambda c^{2}}{8 \pi G}
$$


or then we write

$$
\Lambda=\frac{8 \pi G}{c^{2}} \rho_{\Lambda},
$$

where the pressure is $p<0$ (anti-gravity) and $\Lambda>0$, which represents a dS space having positive curvature given by $\Lambda$ (vacuum energy) as we must have $\rho_{\Lambda}>0$.

Although Eq. (53) is already known, the novelty here is that it was obtained by taking into account the vacuum of SSR associated with an invariant minimum speed $V$ that leads naturally to the EOS of vacuum $\left(p=-\rho_{\Lambda}\right)$ and thus the cosmological constant $\Lambda$, i.e. $\Lambda$ and $\rho_{\Lambda}$ have origin in the spacetime of $\operatorname{SSR}(V)$.

\subsection{The negative scalar curvature (attractive gravity) and its connection with the average distribution of matter in the universe}

In this section, we show how the scalar curvature of the universe $(R)$ is connected to its density of matter, generating an attractive gravity that try to oppose its accelerated expansion due to $\Lambda$ in the current universe. In order to do that, we use the well-known Einstein equation without the cosmological constant and only with the presence of a source of matter (gravity) given by the energy-momentum tensor $T_{\mu \nu}$, namely:

$$
R_{\mu \nu}-\frac{1}{2} R g_{\mu \nu}=\frac{8 \pi G}{c^{2}} T_{\mu \nu}
$$

As we are in the cosmological scenario, where we consider the whole universe with its low homogeneous (average) density of matter, the whole universe can be treated as a perfect fluid, including all kinds of matter and also the dark and vacuum energies. In this sense, the universe is isotropic as it is a perfect fluid with the same pressure $p$ in all directions, so that the tensor $T_{\mu \nu}$ is given by a diagonal matrix. However, in this case, only the effects of gravity (matter) should be taken into account in the energy-momentum tensor of perfect fluid, where we should make a certain approximation such that the effects of matter prevail.

We should realize that the energy-momentum tensor of perfect fluid of SSR $\left(T_{\mu \nu}^{\mathrm{ssr}}\right)$ given in Eq. (46) contains matter (gravity regime) and also vacuum energy due to the cosmological constant (anti-gravity regime) whose EOS is obtained just in the vacuum approximation $v \approx V$. So now it is easy to conclude that when making the approximation $v \gg V$ in the tensor of SSR [Eq. (46)], the vacuum effects are neglected and the well-known energy-momentum tensor of perfect fluid for representing just matter diluted uniformly in the whole space takes place, so that we write the following equation:

$$
R_{\mu \nu}-\frac{1}{2} R g_{\mu \nu}=\frac{8 \pi G}{c^{2}} T_{\mu \nu, v \gg V}^{\mathrm{ssr}},
$$

where $T_{\mu \nu, v \gg V}^{\mathrm{ssr}} \approx T_{\mu \nu}$, i.e. the energy-momentum tensor of perfect fluid of General Relativity (GR) theory is recovered. 
According to Eq. (46), we can calculate the trace $T$ of $T_{\mu \nu}^{\mathrm{ssr}}$, namely:

$$
T=T_{\mu}^{\mu}=(p+\rho) \mathcal{U}^{\mu} \mathcal{U}_{\mu}-p \delta_{\mu}^{\mu},
$$

from where we obtain

$$
T=(p+\rho)\left(1-\frac{V^{2}}{v^{2}}\right)-4 p,
$$

where we find $\mathcal{U}^{\mu} \mathcal{U}_{\mu}=\mathcal{U}_{\mu} \mathcal{U}^{\mu}=\left(1-V^{2} / v^{2}\right)$ and $\delta_{\mu}^{\mu}=4$.

It is interesting to note that, when making $v \gg V$ in Eq. (57), we obtain the matter and radiation regime far from the vacuum regime, so that we recover the trace $T$ of the well-known energy-momentum tensor of perfect fluid of GR $\left(T_{\mu \nu}\right)$, namely:

$$
T=\rho-3 p,
$$

where $T=0$ for the case of the electromagnetic field. Here we are only interested in the case of pure matter with $p=0$, so that $T=\rho=\rho_{m}$.

Let us now obtain Eq. (54) [or Eq. (55)] in the form of its mixed components, as follows:

$$
R_{\mu}^{\nu}-\frac{1}{2} \delta_{\mu}^{\nu} R=\frac{8 \pi G}{c^{2}} T_{\mu}^{\nu}
$$

By making $\mu=\nu$ in Eq. (59) above and knowing that $\delta_{\mu}^{\mu}=4$, we get

$$
R=-\frac{8 \pi G}{c^{2}} T
$$

where $R$ is the curvature of the whole universe that presents an average density of matter given by $T=\rho_{\text {matter }}=\rho_{m}$. So, finally we obtain

$$
R=-\frac{8 \pi G}{c^{2}} \rho_{m}
$$

As the trace of any energy-momentum tensor is always positive or null, in the present case where we just consider the universe filled by matter (including all kinds of attractive matter), we must have $T=\rho_{m}>0$, so that we find a negative scalar curvature $(R<0)$, which indicates that the presence of matter (attractive gravity) generates a constant negative curvature for representing a maximal space with negative curvature, $\frac{19}{19}$ contrary to $\Lambda>0$ [Eq. (53)] that works like a positive curvature (cosmological anti-gravity) that contraposes $R<0$.

\subsection{The flat space: the $\Lambda C D M$ model}

It is known that the $\Lambda \mathrm{CDM}$ model assumes that the universe is composed of photons, neutrinos, ordinary matter (baryons, electrons) and cold (non-relativistic) dark matter (CDM), which only interacts gravitationally, plus dark energy (or a vacuum energy), which is responsible for the observed acceleration in the Hubble expansion. Dark energy is assumed to take the form of a constant vacuum energy density referred to the cosmological constant $(\Lambda)$ as shown in Eq. (50) by SSR. The 
Standard $\Lambda$ CDM Model with such 6 parameters further imposes the constraint that space is flat (Euclidean). However, we should stress that its exact shape is still a matter of debate in physical cosmology, but experimental data from various independent sources (WMAP, BOOMERanG and Planck) confirm that the observable universe is flat according to $\Lambda \mathrm{CDM}$ or at least almost flat. It was found about $0.4 \%$ margin of error whose origin is not clear yet. High-resolution maps of the cosmic microwave background (CMB) radiation has confirmed a flat universe, ${ }^{25}$ but the presence of such minor errors is still inevitable.

The universe has about $4 \%$ of visible matter (vm) given by the baryonic matter, also including electrons since the quantity of anti-matter is minimal and can be neglected. This $4 \%$ is composed of only about $0.4 \%$ of stars plus $3.6 \%$ of interstellar matter (gases), i.e. we write $\Omega_{\mathrm{vm}} \approx 0.04$. It has about $23 \%$ of $\mathrm{CDM}$, i.e. $\Omega_{\mathrm{CDM}} \approx$ 0.23 and finally with about $73 \%$ of dark energy, i.e. $\Omega_{\Lambda} \approx 0.73$, so that the whole universe has $\Omega=\Omega_{m}+\Omega_{\Lambda}=1$ (a flat universe), where $\Omega_{m}=\Omega_{\mathrm{vm}}+\Omega_{\mathrm{CDM}}$ represents the result of all kinds of attractive matter (PS: here the effects of neutrinos and photons $(T=0)$ are so minimal that they do not affect our results). Therefore, we can write Eq. (61) by considering all the components of attractive matter, namely:

$$
R=-\frac{8 \pi G}{c^{2}} \rho_{m}=-\frac{8 \pi G}{c^{2}}\left(\rho_{\mathrm{vm}}+\rho_{\mathrm{CDM}}\right) .
$$

According to SSR given in the regime of vacuum $(v \approx V)$ whose energy governs about $73 \%$ of the universe, i.e. $\Omega_{\Lambda} \approx 0.73$, we have already found

$$
\Lambda=\frac{8 \pi G}{c^{2}} \rho_{\Lambda}
$$

Finally, by adding the effects of scalar curvatures of repulsive $(\Lambda)$ and attractive (vm plus CDM) gravity given in Eqs. (63) and (62), i.e. $\Lambda+R$, we find the effective scalar curvature $R_{\text {eff }}=\Lambda+R$, namely:

$$
R_{\mathrm{eff}}=R_{\mathrm{vm}+\Lambda+\mathrm{CDM}}=\frac{8 \pi G}{c^{2}}\left[\rho_{\Lambda}-\left(\rho_{\mathrm{vm}}+\rho_{\mathrm{CDM}}\right)\right]
$$

As the universe is flat, i.e. $\Omega=1$, then $\Lambda$ CDM model becomes consistent with Eq. (64) only if the effective scalar curvature $R_{\mathrm{eff}}=R_{\mathrm{vm}+\Lambda+\mathrm{CDM}}=0$, so that we find

$$
\rho_{\Lambda}-\left(\rho_{\mathrm{vm}}+\rho_{\mathrm{CDM}}\right)=0,
$$

or simply $\rho_{\Lambda}=\rho_{m}$, which is the cosmic coincidence for the current universe, leading to an Euclidean universe as $R_{\text {eff }}=0$.

In a previous work, $\frac{16}{,}$ according to SSR theory, it was demonstrated that the constant $\Lambda$ is in fact a cosmological parameter that depends on the Hubble radius $R_{H}$, being written as follows:

$$
\Lambda=\frac{6 c^{2}}{R_{H}^{2}} .
$$

As $R_{H} \sim 10^{26} \mathrm{~m}$, we find $\Lambda \sim 10^{-35} \mathrm{~s}^{-2}$. This positive tiny order of magnitude of $\Lambda$ is in agreement with observations. 
By substituting Eq. (66) in Eq. (63), we obtain the vacuum energy density $\rho_{\Lambda}$ depending on the Hubble radius, namely:

$$
\rho_{\Lambda}=\frac{3 c^{4}}{4 \pi G R_{H}^{2}} .
$$

On the other hand, we obtain the total average density of matter $\rho_{m}$ for the spherical universe with total mass $M$ and Hubble radius $R_{H}$ as being

$$
\rho_{m}=\frac{3 M}{4 \pi R_{H}^{3}} .
$$

Due to the cosmic coincidence that leads to a flat universe as $\rho_{m}=\rho_{\Lambda}$, thus we write

$$
\frac{3 M}{4 \pi R_{H}^{3}}=\frac{3 c^{4}}{4 \pi G R_{H}^{2}} \sim 10^{-29} \mathrm{~g} / \mathrm{cm}^{3},
$$

which indeed occurs for the current Hubble radius $R_{H} \sim 10^{26} \mathrm{~m}$, where $M=$ $M_{\mathrm{vm}}+M_{\mathrm{CDM}} \sim 10^{54} \mathrm{~kg}$.

Here we should note that, as $\rho_{\Lambda} \propto R_{H}^{-2}$ decreases slower than $\rho_{m} \propto R_{H}^{-3}$ with the Hubble radius expansion, the cosmological anti-gravity of expansion will overcome the attractive gravity among the galaxies, i.e. $\rho_{\Lambda}>\rho_{m}$ in the future. Therefore SSR predicts that the universe will no longer be flat in the future and it will have an increasing of positive curvature governed by accelerated expansion, since $R_{\text {eff }}=\Lambda+R>0$. So, in the future, SSR theory shows that the universe will be governed by a dS scenario. This is the reason why we are interested in making a GUP in a dS scenario $(\phi<0), \frac{19}{19}$ given for the approximation of a weak anti-gravity, i.e. $R_{\text {eff }}=\Lambda-R=\Lambda_{\text {eff }} \geq 0$. In the next section, we will also investigate GUP in a AdS scenario with a negative $\Lambda_{\text {eff }}$, but close to zero.

\section{GUP in dS and AdS Spaces Within SSR Scenario}

The graph in Fig. 2 shows the transition between gravity (AdS spaces) and antigravity (dS spaces).

\subsection{GUP in dS spaces governed by anti-gravity: $-c^{2} \leq \phi \leq 0^{-}$ $\left(R_{\text {eff }} \geq 0\right)$}

From Eq. (18), we have obtained the uncertainty relation in a quasi-flat spacetime by considering the slight deviation on the reduced Planck constant due to the background field at $S_{V}$, i.e. $\hbar(1-\xi)$ with $\xi=V / c$, which leads exactly to

$$
\eta_{\mu \nu} \mathcal{P}^{\mu} \mathcal{X}_{\text {rec }}^{\nu}=\hbar(1-\xi) \text {. }
$$

By multiplying both sides of Eq. (70) by the conformal factor $\Theta$, we obtain

$$
G_{\mu \nu} \mathcal{P}^{\mu} \mathcal{X}_{\mathrm{rec}}^{\nu}=\frac{\hbar(1-\xi)}{\left(1-\frac{V^{2}}{v^{2}}\right)} .
$$




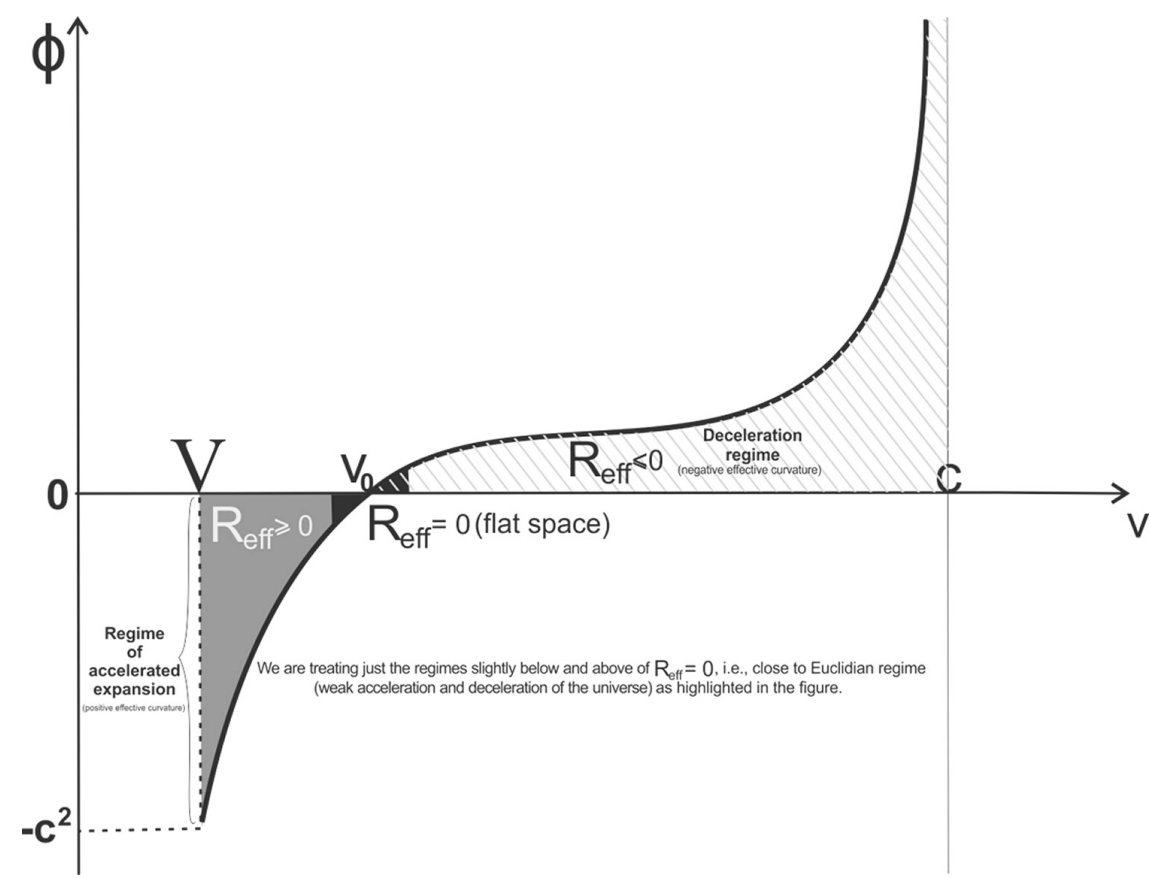

Fig. 2. The graph shows the scalar potential $\phi(v)=c^{2}\left(\sqrt{\frac{1-\frac{V^{2}}{v^{2}}}{1-\frac{v^{2}}{c^{2}}}}-1\right)$. It shows the two phases and their connections with the effective scalar curvature of the universe $\left(R_{\text {eff }}\right)$, namely the effective gravity (right side) with $R_{\text {eff }}<0$ and the effective anti-gravity (left site) with $R_{\text {eff }}>0$. We see the barrier at the right side, representing the relativistic limit close to the speed of light $c$ with $\phi \rightarrow \infty$, and the barrier at the left side representing the quantum (anti-gravitational) limit only described by SSR (minimum speed $V$ with $\phi=-c^{2}$ ). The intermediary region $V \ll v \ll c$ is the Newtonian regime, which represents a quasi-flat space with a quasi-zero scalar effective curvature, i.e. $R_{\mathrm{eff}} \approx 0$ as matter and vacuum energy are in equal footing, representing the state of the current universe. The speed $v=v_{0}=\sqrt{c V}$ is the point of phase transition where the space is flat $\left(R_{\mathrm{eff}}=0\right.$ with $\left.\phi=0\right)$ below which $\left(v<v_{0}\right)$ the repulsive vacuum effect takes place as $R_{\text {eff }}>0(\phi<0)$, and above which $\left(v>v_{0}\right)$ the attractive gravity (matter) takes place as $R_{\text {eff }}<0(\phi>0)$.

By expanding the second side of Eq. (71), we write

$$
G_{\mu \nu} \mathcal{P}^{\mu} \mathcal{X}_{\mathrm{rec}}^{\nu}=\hbar(1-\xi)\left[1+\frac{V^{2}}{v^{2}}+\frac{V^{4}}{2 ! v^{4}}+\cdots\right] .
$$

Let us now consider the approximation for a slightly repulsive potential $\phi \approx 0^{-}$, so that $v$ is slightly smaller than $v_{0}(\gg V)$, where $\phi(V)=-c^{2}$ is the most repulsive potential (Fig. 2). In doing this in Eq. (72), we write

$$
G_{\mu \nu} \mathcal{P}^{\mu} \mathcal{X}_{\text {rec }}^{\nu} \approx \hbar(1-\xi)\left[1+\frac{V^{2}}{v^{2}}\right]
$$

Equation (73) can be written in its equivalent form of weakly repulsive potential, namely: 


$$
G_{\mu \nu} \mathcal{P}^{\mu} \mathcal{X}_{\mathrm{rec}}^{\nu} \approx \hbar(1-\xi)\left(1-\frac{2 \phi}{c^{2}}\right)
$$

where $\phi \leq 0^{-}$(see Fig. 2).

We already know that the momentum $P$ is given with respect to $S_{V}$, but it represents an uncertainty $\Delta p$ at any Galilean reference frame $S$, i.e. we write $P \equiv$ $(\Delta p)_{S}=\Delta p \cdot .^{22}$

As we are interested to investigate $\mathrm{dS}$ spaces, where $V<v \leq v_{0}$ (Fig. 2), let us write the uncertainty $\Delta p$ only for the repulsive sector of gravity (anti-gravity), namely:

$$
\Delta p \equiv P=m_{0} v \sqrt{1-\frac{V^{2}}{v^{2}}}=m_{0} v\left(1+\frac{\phi(r)}{c^{2}}\right),
$$

where $V<v \leq v_{0}$ for $-c^{2}<\phi \leq 0^{-}$.

By considering the slightly repulsive potential $\phi \leq 0^{-}$, where $v \leq v_{0}$ or then $v \approx v_{0}^{-}$, so we make the approximation in Eq. (75) as follows:

$$
\Delta p \approx m_{0} v_{0}\left(1+\frac{\phi(r)}{c^{2}}\right)=p_{0}\left(1+\frac{\phi(r)}{c^{2}}\right)
$$

where we simply have made $m_{0} v \approx m_{0} v_{0}=m_{0} \sqrt{c V}=p_{0}$, since $v \approx v_{0}^{-}$.

From Eq. (76), we get

$$
\Delta p-p_{0} \approx \frac{p_{0} \phi(r)}{c^{2}}
$$

from where we obtain

$$
\frac{2 \phi(r)}{c^{2}} \approx \frac{2\left(\Delta p-p_{0}\right)}{p_{0}}
$$

By substituting Eq. (78) in Eq. (74), we finally get

$$
G_{\mu \nu} \mathcal{P}^{\mu} \mathcal{X}_{\mathrm{rec}}^{\nu} \approx \hbar(1-\xi)\left[1-\frac{2\left(\Delta p-p_{0}\right)}{p_{0}}\right],
$$

where we realize that $\Delta p<p_{0}$ for $\mathrm{dS}$ spaces with small positive cosmological constants (weak anti-gravity).

Equation (79) represents the modified uncertainty relation in the presence of a weakly repulsive anti-gravity on the surface of a sphere of dark energy with radius $r$.

\subsection{GUP in AdS spaces governed by gravity: $0^{+} \leq \phi<\infty$ $\left(\boldsymbol{R}_{\text {eff }} \leq \mathbf{0}\right)$}

First of all, it is important to perceive that the well-known conformal metric $\left(G_{\mu \nu}\right)_{\mathrm{dS}}[\mathrm{Eq} .(71)]$ given in dS spaces can be transformed into another conformal metric $\left(G_{\mu \nu}\right)$ AdS given in AdS spaces only by performing the reciprocal form of 
$\left(G_{\mu \nu}\right)_{\mathrm{dS}}=G_{\mu \nu}$, i.e. we have to calculate the reciprocal metric $G_{\mu \nu, \text { rec }}$ in order to obtain $\left(G_{\mu \nu}\right)$ AdS. So we write

$$
\left(G_{\mu \nu}\right)_{\mathrm{AdS}}=G_{\mu \nu, \mathrm{rec}}=\frac{1}{\left(1-\frac{V^{2}}{v_{\mathrm{rec}}^{2}}\right)} \eta_{\mu \nu} .
$$

As we already know that $v_{\text {rec }}=v_{0}^{2} / v=c V / v$, Eq. (80) is written as

$$
\left(G_{\mu \nu}\right)_{\mathrm{AdS}}=G_{\mu \nu, \mathrm{rec}}=\frac{1}{\left(1-\frac{v^{2}}{c^{2}}\right)} \eta_{\mu \nu} .
$$

Thus Eq. (71) is transformed into its reciprocal form (AdS spaces), namely:

$$
G_{\mu \nu, \mathrm{rec}} \mathcal{P}^{\mu} \mathcal{X}_{\mathrm{rec}}^{\nu}=\hbar(1-\xi)\left[1+\frac{v^{2}}{c^{2}}+\frac{v^{4}}{2 ! c^{4}}+\cdots\right]
$$

which represents the attractive potential $(\phi>0)$ as we are in the Lorentz sector, i.e. $v_{0}<v<c$ (Fig. 2).

Let us now consider the approximation for a weakly attractive potential $\phi \approx 0^{+}$, so that $v$ is not much larger than $v_{0}$ (Fig. 2). In doing this in Eq. (82), we write

$$
G_{\mu \nu, \mathrm{rec}} \mathcal{P}^{\mu} \mathcal{X}_{\mathrm{rec}}^{\nu}=\hbar(1-\xi)\left[1+\frac{v^{2}}{c^{2}}\right] .
$$

Equation (83) can now be written in its equivalent form of weakly attractive potential as follows:

$$
G_{\mu \nu, \mathrm{rec}} \mathcal{P}^{\mu} \mathcal{X}_{\text {rec }}^{\nu}=\hbar(1-\xi)\left[1+\frac{2 \phi}{c^{2}}\right]
$$

where we must have $\phi \geq 0^{+}$.

As we are now interested to investigate AdS spaces, where $v_{0} \leq v<c$ (Fig. 2), let us write the uncertainty $\Delta p$ only for the attractive sector of gravity (Lorentz sector), namely:

$$
\Delta p \equiv P=\frac{m_{0} v}{\sqrt{1-\frac{v^{2}}{c^{2}}}}=m_{0} v\left(1+\frac{\phi(r)}{c^{2}}\right)
$$

where $0<\phi<\infty$.

By considering a weakly attractive potential $\phi \geq 0^{+}$, where $v \geq v_{0}$ or then $v \approx v_{0}^{+}$, so we make the approximation in Eq. (85) as follows:

$$
\Delta p \approx p_{0}\left(1+\frac{\phi(r)}{c^{2}}\right)
$$

where $p_{0}=m_{0} v_{0}$.

From Eq. (86), we get

$$
\Delta p-p_{0} \approx \frac{p_{0} \phi(r)}{c^{2}}
$$


from where we obtain

$$
\frac{2 \phi(r)}{c^{2}} \approx \frac{2\left(\Delta p-p_{0}\right)}{p_{0}}
$$

By substituting Eq. (88) in Eq. (84), we finally obtain

$$
G_{\mu \nu, \mathrm{rec}} \mathcal{P}^{\mu} \mathcal{X}_{\mathrm{rec}}^{\nu} \approx \hbar(1-\xi)\left[1+\frac{2\left(\Delta p-p_{0}\right)}{p_{0}}\right],
$$

where we have $\Delta p>p_{0}$ and $G_{\mu \nu \text {,rec }}=\left(G_{\mu \nu}\right)_{\text {AdS }}$ for AdS spaces with weak gravity.

Equation (89) represents the modified uncertainty relation in the presence of a weakly attractive gravity on the surface of a sphere dominated by matter and with radius $r$.

Here it is interesting to mention a recent Ref. 26 that deals with GUP by modifying the Heisenberg principle in presence of a gravitational field. The authors have found a deformed uncertainty relation which reminds Eq. (89), since $\Delta p$ also appears in the second side of their relation by modifying $\hbar$, thus leading to an effective reduced Planck constant. ${ }^{26}$

\section{Acknowledgments}

I am grateful to the heated discussions with my colleagues Fernando Antônio da Silva, Rodrigo Francisco dos Santos and Antônio Carlos Amaro de Faria Jr.

This work is dedicated to the memory of Albert Einstein due to the 100th anniversary of Sobral's eclipse and the proof of the theory of General Relativity. His obstinate attempts of unification indicated that it was needed and possible to obtain the geometrization of the Uncertainty Principle, since he never accepted the indetermination of Quantum Mechanics and sought incessantly for the objective reality. This would lead to deeper scientific and philosophical reflections of the experimental results, which his contemporaries have dispensed. However, the present research, already introduced in other quoted articles, attempts to reestablish the deterministic order in Nature by validating his efforts. After all, it was written on the portal of Plato's Academy: "Whoever is not geometer does not enter."

\section{References}

1. R. Bluhm, Lect. Notes Phys. 702, 191 (2006).

2. R. Jackiw and A. Kostelecky, Phys. Rev. Lett. 82, 3572 (1999).

3. S. M. Carroll, G. B. Field and R. Jackiw, Phys. Rev. D 41, 1231 (1990).

4. V. A. Kostelecky and N. Russell, Rev. Mod. Phys. 83, 11 (2011).

5. V. A. Kostelecky and M. Mewes, Phys. Rev. D 66, 056005 (2002).

6. T. Jacobson, S. Liberati and D. Mattingly, Phys. Rev. D 67, 124011 (2003).

7. J. J. Wei and X. Feng Wu, Astrophys. J. 851, 127 (2017).

8. C. A. G. Almeida, M. A. Anacleto, F. A. Brito, E. Passos and J. R. L. Santos, arXiv:hep-th/1712.00995.

9. M. Schreck, arXiv:hep-th/1711.11167.

10. T. P. Netto, arXiv:hep-th/1711.05193. 
11. T. Prudencio, L. S. Amorim, H. Belich and H. L. C. Louzada, arXiv:physics.gen$\mathrm{ph} / 1706.04456$.

12. L. Nogués et al., arXiv:astro-ph/1710.08342.

13. G. Lambiase and F. Scardigli, arXiv:hep-th/1709.00637.

14. J. Magueijo and L. Smolin, Phys. Rev. Lett. 88, 190403 (2002).

15. G. A. Camelia, Nature 418, 34 (2002).

16. C. Nassif, Int. J. Mod. Phys. D 25, 1650096 (2016).

17. C. Nassif, Gen. Relat. Gravit. 47, 1 (2015).

18. C. Nassif, Pramana-J. Phys. 71, 1 (2008).

19. C. Nassif, R. F. dos Santos and A. C. Amaro de Faria Jr., Int. J. Mod. Phys. D 27 1850011 (2018).

20. C. Nassif, A. C. Amaro de Faria Jr. and Rodrigo Francisco dos Santos, Mod. Phys. Lett. A 33, 1850148 (2018).

21. C. Nassif, Int. J. Mod. Phys. D 19, 539 (2010).

22. C. Nassif, Int. J. Mod. Phys. D 21, 1 (2012).

23. D. Marolf, Phys. Rev. Lett. 114, 031104 (2015).

24. C. Nassif, R. F. dos Santos and A. C. Amaro Junior, Phys. Dark Universe 22, 116 (2018).

25. P. de Bernardis, et al., Nature 404, 955 (2000).

26. A. Alonso-Serrano, M. P. Dabrowski and H. Gohar, Phys. Rev. D 97, 044029 (2018). 